\title{
Unexplained Persistent Dyspnea in a Young Woman With Eosinophilic Angiocentric Fibrosis
}

\author{
Woo-Jin Kim MD, Yu-Il Kim MD, Ji-Eun Kim MD, Young-Hwan Choi MD, \\ Hyong-Ho Cho MD, Yoo-Duk Choi MD, Hyun-Ju Seon MD, and Sung-Ho Yoon MD
}

\begin{abstract}
Eosinophilic angiocentric fibrosis (EAF) is a rare inflammatory disease that primarily involves the nose and sinuses. Involvement of the eye orbit and larynx has also been described. However, it is very rare for this disease to involve the lower respiratory tract and cause dyspnea. We describe a rare case of EAF involving the lower respiratory tract with airway narrowing. A 29-year-old female with a 7-year history of nasal obstruction presented with unexplained persistent dyspnea. EAF was diagnosed via endoscopic biopsy of an irregular mucosal lesion in the posterior wall of the right maxillary sinus. Chest computed tomography and bronchoscopy showed a diffuse inflammatory narrowing of the airway in the tracheobronchial trees. EAF can affect lower respiratory tracts with airway narrowing that can be characterized by dyspnea. We must consider narrowing of the lower respiratory tracts in patients with EAF complaining of unexplained persistent dyspnea. Key words: dyspnea; eosinophilic angiocentric fibrosis; airway narrowing. [Respir Care 2014;59(5):e72-e76. (C 2014 Daedalus Enterprises]
\end{abstract}

\section{Introduction}

Eosinophilic angiocentric fibrosis (EAF) is an unusual fibrotic condition of unknown etiology that most commonly affects the sinonasal cavity. It was first described in 1983 by Holmes and Panje, ${ }^{1}$ who reported a case with recurring intranasal mass. Other than the nose and sinuses, the other structures such as the eye orbit, gums, larynx, and upper trachea can be involved as well. ${ }^{2-5}$ However, lower airway involvement is very rare, and was reported for the first time in 2011 by Deshpande et al. ${ }^{6}$

Drs Woo-Jin Kim, Yu-Il Kim, Ji-Eun Kim, and Young-Hwan Choi are affiliated with the Department of Internal Medicine, Dr Cho is affiliated with the Department of Otolaryngology-Head and Neck Surgery, Dr YooDuk Choi is affiliated with the Department of Pathology, and Dr Seon is affiliated with the Department of Radiology, Chonnam National University Hospital, Gwangju, South Korea. Dr Yoon is affiliated with the Department of Internal Medicine, Chosun University, College of Medicine, Gwangju, South Korea.

Correspondence: Dr Yu-Il Kim, Division of Pulmonology, Department of Internal Medicine, Chonnam National University Hospital, 42, Jebongro, Donggu, Gwangju 501-757, South Korea. E-mail: kyionly@ chonnam. ac.kr.

DOI: $10.4187 /$ respcare. 02645
The precise etiology of EAF still remains unclear. Atopy, immunologic disease, previous surgery, and trauma have been suggested to be predisposing factors, but the evidence for any of these origins is weak. 7,8

To the best of our knowledge, there are few case reports that show diffuse inflammatory narrowing of the tracheobronchial tree in patients with EAF. In this report, we describe a rare case of EAF with diffuse narrowing of the lower respiratory tract in a patient presenting with dyspnea.

\section{Case Report}

A 29-y-old female presented with a 7-y history of nasal stuffiness and frequent productive cough and dyspnea. The dyspnea was not paroxysmal. Breathlessness had gradually increased over $4 \mathrm{y}$. It was described as persistent for the previous year, but she did not have fever, chills, arthralgia, or hemoptysis. She never smoked. An ostiomeatal unit (OMU) computed tomography (CT) scan revealed chronic sinusitis involving the right maxillary sinus and obstruction of the right OMU (Fig. 1A). Nasopharyngoscopy showed purulent nasal discharge with diffuse mucosal swelling. She underwent endoscopic biopsy of an irregular mucosal lesion in the posterior wall of her right maxillary sinus. This lesion of the sinuses suggested dif- 


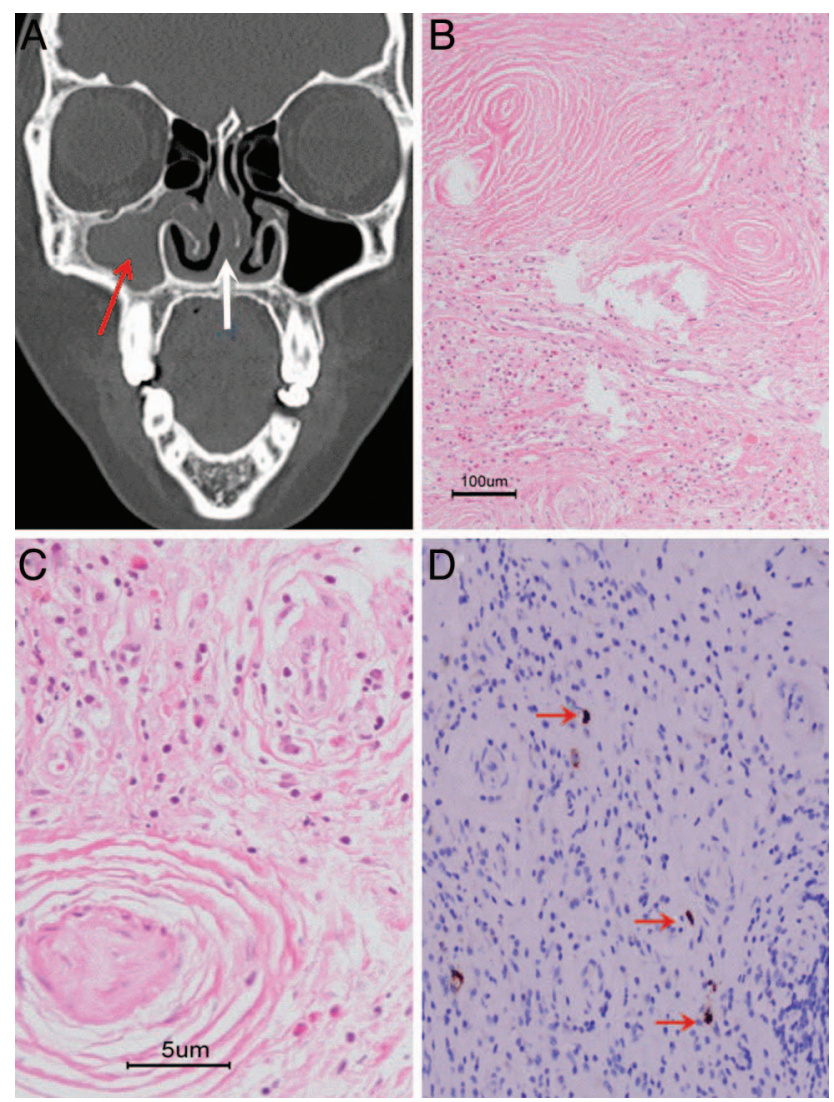

Fig. 1. Ostiomeatal unit computed tomography (CT) and biopsy of a mucosal lesion in the right maxillary sinus. A: A CT image showed chronic sinusitis involving the right maxillary sinus (red arrow), obstruction of the right $\mathrm{OMU}$, and a deviation of the nasal septum to the left side (white arrow). The biopsy from the mucosal lesion in the right maxillary sinus was compatible with EAF, a distinctive perivascular fibrosis with an 'onion-skin-type' appearance (hematoxylin and eosin [H\&E], $\times 20$; B) and cellular inflammatory cells composed of eosinophils, lymphocytes, plasma cells, neutrophils, and histiocytes (H\&E, $\times 200 ; C)$. D: On IgG4 immunohistochemical staining of the nasal biopsy, a few cells (arrows, 5-6 per highpower field) showed immunoreactivity toward lgG4 $(\times 200)$.

ferential diagnoses such as Wegener's granulomatosis with polyangiitis, Churg-Strauss syndrome (CSS), Kimura disease, and granuloma faciale from EAF. To exclude these mimickers laboratory investigations and histopathological assessment of the biopsy tissue were performed. The biopsy result was characterized by a perivascular eosinophilrich inflammatory infiltrate and fibrosis leading to a characteristic whirling, onion-skin-type pattern that is pathognomonic for EAF (see Fig. 1, B and C). On immunohistochemical staining of the nasal biopsy, a few cells (red arrows, 5 6 cells per high-power field) showed an immunoreactivity toward immunoglobulin G4 (IgG4) (see Fig. 1D).

Laboratory investigations for antinuclear antibodies, anti-neutrophil cytoplasmic antibodies (ANCAs), cytoplasmic ANCAs, perinuclear ANCAs, urinalysis (including proteinuria and hematuria), and C-reactive protein were all negative. The only abnormal finding was a mildly elevated erythrocyte sedimentation rate $(32 \mathrm{~mm} / \mathrm{h})$. Ig levels (IgG, $1280 \mathrm{mg} / \mathrm{dL} ; \mathrm{IgE}, 29.6 \mathrm{IU} / \mathrm{mL}$ ) and eosinophil count (1.7\%) were within the normal range. A pulmonary function test showed a primarily moderate obstructive pattern with a mild restrictive pattern $\left(\mathrm{FEV}_{1}, 1.62 \mathrm{~L}[58 \%\right.$ predicted]; FVC, 2.7 L [78\% predicted]; $\mathrm{FEV}_{1} / \mathrm{FVC}$, 0.60; residual volume, $0.82 \mathrm{~L}$ [62\% predicted]; total lung capacity, 3.52 L [78\%]), suggesting chronic respiratory diseases such as COPD, bronchiectasis, or others. The diffusing capacity of the lung for carbon monoxide was within the normal range $(18.4 \mathrm{~mL} / \mathrm{min} / \mathrm{mm} \mathrm{Hg}, 87 \%$ predicted). There was no significant reversibility of the airway obstructive pattern, and the patient had no history of allergies. To palliate her symptoms, she was prescribed bronchodilators. However, she still presented with persistent dyspnea with wheezing on mild exertion. A chest $\mathrm{CT}$ scan was performed to further evaluate her symptoms. The CT scan revealed diffuse, smooth, concentric tracheal wall thickening as well as thickening of the bilateral main, lobar, segmental, and some subsegmental bronchi, with luminal narrowing of the tracheobronchial trees (Fig. 2, A and B). Bronchoscopy revealed overall erythematous mucosal thickening and narrowing of entire tracheobronchial trees, which were thought to be related to a chronic inflammatory condition. A single bronchoscopic biopsy was performed at the carina because of severe dyspnea and desaturation during bronchoscopy. It showed squamous metaplasia with neutrophils, which is consistent with chronic inflammation (Fig. 3). Bronchial washing was negative for bacteria, fungus, and tuberculosis. Surgical biopsy for lesions of the lower respiratory tract was recommended to confirm the tracheobronchial involvement of the EAF. However, the patient refused to undergo surgical biopsy of the lower respiratory airways. The patient was subsequently treated with a systemic steroid (prednisone) and bronchodilators (fluticasone/salmeterol inhaler and doxofylline) for 3 months. Mucolytic agents, including erdosteine, were also prescribed. Nasal and respiratory symptoms have somewhat waxed and waned over time. Systemic steroid treatment with dose escalation was effective when nasal and respiratory symptoms increased. A follow-up chest CT scan showed more improvement of tracheobronchial narrowing (see Fig. 2, C and D). We measured the dimensions of the airways at the same anatomic locations before and after treatment. The improvement of airway involvement was evaluated as an increase in inner luminal diameter and a decrease in airway wall thickness. The patient's tracheal dimensions were measured at the level of the aortic arch. The tracheal inner diameter increased from 13.9 to $15.1 \mathrm{~mm}$, and the tracheal wall thickness decreased from 2.0 to $1.8 \mathrm{~mm}$ after treatment. The diameters of the segmental bronchi also appeared to increase. Airway measurements are detailed in 

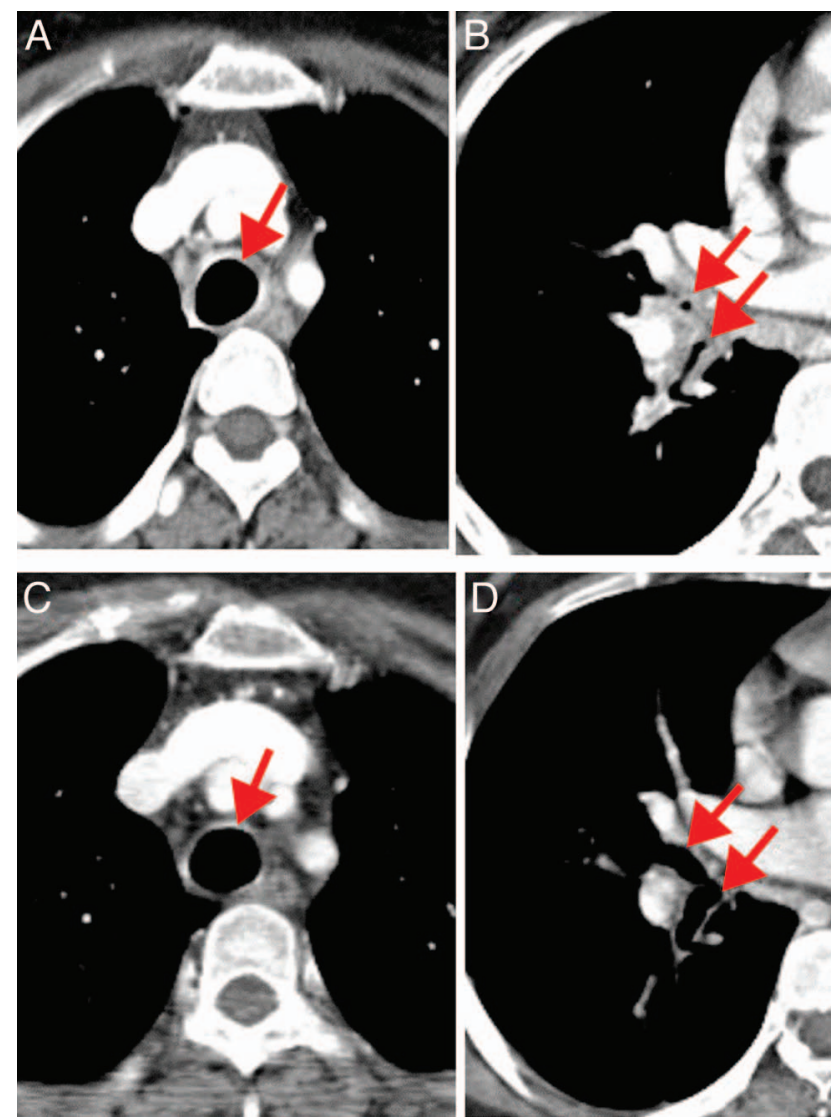

Fig. 2. Chest computed tomographic images at baseline and at 3 months after treatment initiation. A and B: Initial images showed a diffuse wall thickening with luminal narrowing of the trachea (arrow at left) and bronchial trees (arrows at right). C and D: Three months after treatment, the tracheal (red arrow at left) and bronchial (arrows at right) wall thicknesses had decreased and the luminal diameters had increased.

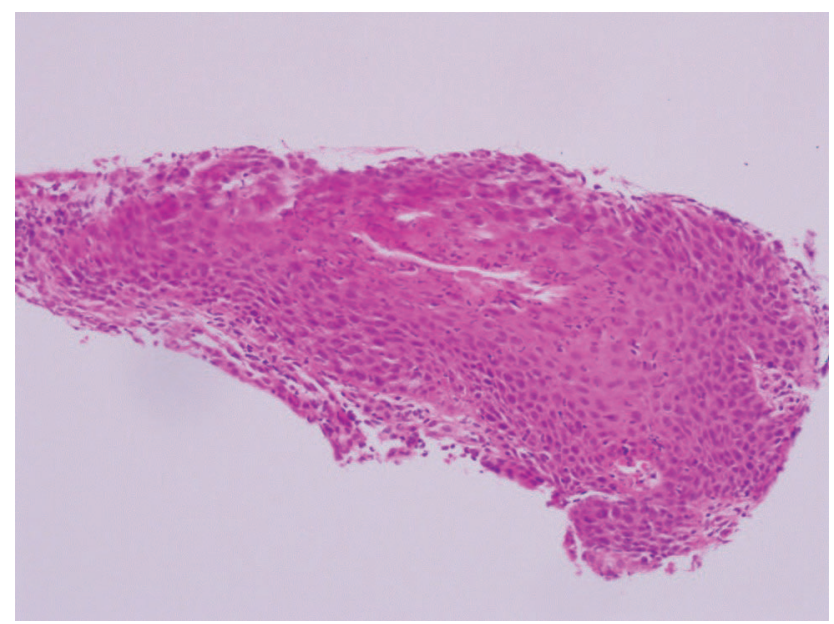

Fig. 3. Bronchoscopic mucosal biopsy from the carina revealed squamous metaplasia with neutrophil infiltration (hematoxylin and eosin, $\times 200)$. our previous publication. ${ }^{9}$ These measurements suggest that some of the improvement in dyspnea may be linked to improved airway dimensions. However, bronchodilator treatment has been maintained to palliate her symptoms because of continued exertional dyspnea.

\section{Discussion}

EAF is a rare inflammatory disease, primarily involving the orbit and upper respiratory tract, particularly the sinonasal cavity. ${ }^{4}$ It was first described in 1983 by Holmes and Panje. ${ }^{1}$ EAF has distinct histological features characterized by a perivascular eosinophil-rich inflammatory infiltrate and fibrosis with a whirling, onion-skin-type pattern. ${ }^{1}$ It presents as a slowly progressive upper airway obstruction in association with a submucosal inflammatory fibrosing lesion. ${ }^{4}$

There are few case reports that show EAF involvement of the trachea and lower respiratory tract. Deshpande et al ${ }^{6}$ reported a 63-year-old man with EAF who had mediastinal, hilar, and axillary lymphadenopathies with extensive ground-glass pulmonary opacities and bronchial wall thickening. Seven years later, they detected a worsening in the patient's pulmonary lesions, an increase in the size of the lymph nodes, and a polypoid lesion from the bronchial wall. In this case, the patient presented with chronic dyspnea and diffuse airway wall thickening that was comparable to the previous report. However, we did not see any mediastinal/hilar lymphadenopathies or parenchymal lesions in our case. This suggests that the airway thickening of the lower respiratory tract can develop as a result of EAF involvement. However, patterns of respiratory system involvement could be variable and could change over time. Chest CT scanning or bronchoscopy can be helpful for evaluating the involvement of the tracheobronchial tree in a patient with EAF who complains of unexplained persistent dyspnea or respiratory symptoms. Long-term follow-up is necessary because of the possibility of progression.

The etiology and pathogenesis of EAF are unknown, but some studies have suggested that it may be part of the spectrum of IgG4-related systemic diseases. ${ }^{6}$ Deshpande et $\mathrm{l}^{6}$ reported that EAF lesions were associated with large numbers of IgG4-positive plasma cells. However, there is no consensus regarding the minimal numbers of $\mathrm{IgG} 4$ positive cells required for the diagnosis of IgG4-related systemic diseases. ${ }^{6}$ In our case, a few cells showed immunoreactivity for $\operatorname{IgG} 4$ upon staining of the nasal biopsy sample.

EAF has usually been distinguished from some of its mimickers, including Wegener's granulomatosis with polyangiitis, CSS, Kimura disease, granuloma faciale, and angiolymphoid hyperplasia with eosinophilia.6,10 These mimickers have been excluded on the basis of laboratory 


\section{Dyspnea in a Woman With Eosinophilic Angiocentric Fibrosis}

investigation findings and histopathological assessment of biopsy tissue. The histology of EAF is pathognomonic and is characterized by perivascular inflammatory cell infiltration with fibrosis around small vessels, leading to a characteristic onion-skin-type pattern. ${ }^{4}$ Absence of geographic necrosis, necrotizing vasculitis, and granulomatous inflammation excludes granulomatosis with polyangiitis and CSS. ${ }^{2,11}$ Blood test results positive for cytoplasmic and perinuclear ANCAs support the diagnoses of granulomatosis with polyangiitis and CSS, respectively. The absence of dense lymphoid aggregates with prominent germinal centers excludes Kimura disease. ${ }^{2}$ Some authors have suggested an association between EAF and granuloma faciale, a benign cutaneous disease that is characterized by circumscribed plaques and skin nodules with a predilection for the facial region. ${ }^{12,13}$ Granuloma faciale lacks the onion-skin-type pattern of collagen whirling around a central vessel that is characteristic of EAF. There is an opinion that EAF is not an entity that is distinct from Wegener's granulomatosis with polyangiitis and atopy. ${ }^{14} \mathrm{EAF}$ is believed to have an allergic etiology. On the contrary, some cases clearly had no allergic etiology, ${ }^{11}$ and the patient in our case also had no history of allergies. However, we did not perform specific allergic examinations such as allergic skin tests. This is a limitation of our study. Further studies are necessary to define the etiology and pathogenesis of EAF.

Treatment guidelines for EAF also remain unclear; there are no definitive treatment modalities. Surgical resection has been performed for progressive or persistent disease, ${ }^{15}$ and local and systemic corticosteroid therapies have been pursued with minimal clinical resolution of disease..$^{4,7,13,16}$ In our case, the sinonasal lesion was well-controlled by surgical resection and systemic steroids. However, steroid treatment with dose escalation was needed when nasal and respiratory symptoms increased.

Rituximab has been proposed as a reasonable approach to therapy in patients with EAF that is refractory to treatment with glucocorticoids. This suggestion is based on an idea that EAF is part of the spectrum of IgG4-related systemic disease. ${ }^{6}$ Additional studies are needed to clarify its treatment.

Pulmonary function testing showed a mixed pattern, primarily obstructive disorder with mild restrictive pattern. We formed the hypothesis that air-flow obstruction resulted from airway narrowing, as seen in COPD, and that the mild restrictive disease may be nonspecific or may be caused by mild parenchymal damage that can be caused by recurrent respiratory infection. The patient had a history of chronic cough with frequent exacerbations, suggesting recurrent respiratory infections.

Another major limitation of this case is the lack of tissue confirmation from the lower respiratory tract. However, in our opinion, the tracheobronchial involvement in this case was caused by EAF for the following reasons: (1) the pattern of airway involvement was comparable to the previous report; (2) there was no evidence of the other diseases that cause diffuse airway narrowing, such as Wegener's granulomatosis with polyangiitis; and (3) EAF usually has pathologic changes below the mucosal level (at the submucosal level). The lesions lead to thickening of the submucosal connective tissues. However, in this case, tracheobronchial tissue was only obtained from the mucosal level because the patient experienced severe hypoxia during the bronchoscopy. This could be the reason that the pathologic confirmation was not performed with a mucosal biopsy sample from the lower respiratory tract.

EAF is a rare, progressive, fibroinflammatory disease primarily involving the sinonasal tract. However, it can affect lower respiratory tracts with airway narrowing that can be characterized by dyspnea. Unexplained persistent dyspnea in a patient with EAF should prompt the clinician to consider diffuse airway narrowing involving the lower respiratory tract.

\section{ACKNOWLEDGMENTS}

The authors thank Enago for the English language review.

\section{REFERENCES}

1. Holmes DK, Panje WR. Intranasal granuloma faciale. Am J Otolaryngol 1983;4(3):184-186.

2. Jain R, Robblee JV, O'Sullivan-Mejia E, Lea J, Heller A, Faquin WC, et al. Sinonasal eosinophilic angiocentric fibrosis: a report of four cases and review of literature. Head Neck Pathol 2008;2(4): 309-315.

3. Karligkiotis A, Volpi L, Ferreli F, Cerati M, Kagkelari E, Meloni F, et al. Primary orbital eosinophilic angiocentric fibrosis with intranasal extension. Head Neck 2013;36(1):E8-E11.

4. Roberts PF, McCann BG. Eosinophilic angiocentric fibrosis of the upper respiratory tract: a mucosal variant of granuloma faciale? A report of three cases. Histopathology 1985;9(11):1217-1225.

5. Fageeh NA, Mai KT, Odell PF. Eosinophilic angiocentric fibrosis of the subglottic region of the larynx and upper trachea. J Otolaryngol 1996;25(4):276-278.

6. Deshpande V, Khosroshahi A, Nielsen GP, Hamilos DL, Stone JH. Eosinophilic angiocentric fibrosis is a form of IgG4-related systemic disease. Am J Surg Pathol 2011;35(5):701-706.

7. Paun S, Lund VJ, Gallimore A. Nasal fibrosis: long-term follow up of four cases of eosinophilic angiocentric fibrosis. J Laryngol Otol 2005;119(2):119-124.

8. Yang BT, Wang YZ, Wang XY, Wang ZC. Nasal cavity eosinophilic angiocentric fibrosis: CT and MR imaging findings. AJNR Am J Neuroradiol 2011;32(11):2149-2153.

9. Kim YI, Schroeder J, Lynch D, Newell J, Make B, Friedlander A, et al. Gender differences of airway dimensions in anatomically matched sites on CT in smokers. COPD 2011;8(4):285-292.

10. Valenzuela AA, Whitehead KJ, Brown I, Sullivan TJ. Eosinophilic angiocentric fibrosis: an unusual entity producing complete lacrimal duct obstruction. Orbit 2006;25(2):159-161. 


\section{Dyspnea in a Woman With Eosinophilic Angiocentric Fibrosis}

11. Thompson LD, Heffner DK. Sinonasal tract eosinophilic angiocentric fibrosis. A report of three cases. Am J Clin Pathol 2001;115(2):243-248.

12. Marcoval J, Moreno A, Peyr J. Granuloma faciale: a clinicopathological study of 11 cases. J Am Acad Dermatol 2004;51(2):269-273.

13. Yung A, Wachsmuth R, Ramnath R, Merchant W, Myatt AE, Sheehan-Dare R. Eosinophilic angiocentric fibrosis-a rare mucosal variant of granuloma faciale which may present to the dermatologist. Br J Dermatol 2005;152(3):574-576.
14. Loane J, Jaramillo M, Young HA, Kerr KM. Eosinophilic angiocentric fibrosis and Wegener's granulomatosis: a case report and literature review. J Clin Pathol 2001;54(8):640-641.

15. Li Y, Liu H, Han D, Zang H, Wang T, Hu B. Eosinophilic angiocentric fibrosis of the nasal septum. Case Rep Otolaryngol 2013;2013:267285.

16. Leibovitch I, James CL, Wormald PJ, Selva D. Orbital eosinophilic angiocentric fibrosis case report and review of the literature. Ophthalmology 2006;113(1):148-152. 